$(15.4 \%)$. At baseline $32.7 \%$ of participants had weight loss, $19.2 \%$ were at risk for malnutrition, and $13.5 \%$ had an impaired ability to prepare meals. Compared to baseline, at 6-months there was an overall increase in self-rated health status $(\mathrm{p}=.006)$, and increased satisfaction with social activity $(\mathrm{p}=.01)$.

Conclusions: Individuals with an acute illness and at acute nutritional risk reported an increase in self-rated health and social activity while receiving weekly medically-appropriate home-delivered meals for 6 months.

\section{THE RELATIONSHIP BETWEEN SCHISANDRAE CHINENSIS SUPPLEMENTATION AND MUSCLE STRENGTH IN OLDER WOMEN}

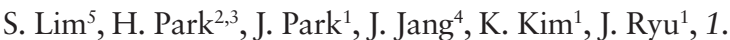
Institute of Taekwondo for Health and Culture, Dong-A University, Busan, Korea (the Republic of), 2. Department of Health Care and Science, Dong-A University, Busan, Korea (the Republic of), 3. Department of Preventive Gerontology, National Center for Geriatrics and Gerontology, Nagoya, Japan, 4. Institute of Convergence Bio-Health, Dong-A University, Busan, Korea (the Republic of), 5. Research Foundation for Industry-Academy Cooperation, Dong-A University, Busan, Korea (the Republic of)

Background: Several studies reported that the direct relaxation effect of schizandra chinesis on animal smooth muscle was more dominant than the endothelium dependent NO pathway in corporal tissue and that activation of $\mathrm{K}^{+}$channels and inhibition of TRPC6 channels could be one mechanism of schizandra chinesis induced relaxation of smooth muscles. The fruit of Schizandra chinensis (SC) is a well-known traditional herb used for pharmacological purposes in Asian countries (e.g., Korea, China and Japan), however, the association of SC sippl. and physical function in older population remains unclear.

Purpose: A controlled, randomized, double-bind trial to evaluate the effect of schizandra chinesis supplementation on skeletal muscle mass and muscle force in older women.

Subjects and Methods: Forty-eight female participants (intervention group, $\mathrm{n}=26$; control group, $\mathrm{n}=22$ ) were included in this study. The intervention group performed ingested two capsules containing either schizandra chinesis or cellulose with every day for 12 weeks breakfast and dinner. The schizandra chinesis capsule contained $250 \mathrm{mg}$ of schizandra chinensis, whereas the placebo capsule contained $250 \mathrm{mg}$ cellulose. Comparison of changes in body composition measured by DXA, muscles quadriceps strength by Biodex, and laboratory tests between the control and intervention groups during 12 weeks.

Results: After intervention, significant group $\times$ time interactions for the strength of quadriceps muscle force were found. Also, significant group $\times$ time interactions for appendicular skeletal muscle mass were found.

Conclusion: Schizandra chinesis supplementation may help to improve the age-related loss of skeletal muscle mass and muscle force in older women.

\section{BIOIMPEDANCE-DERIVED PHASE ANGLE AND MORTALITY AMONG OLDER PEOPLE}

L. Genton ${ }^{4}$, K. Norman 2 , A. Spoerri ${ }^{3}$, C. Pichard ${ }^{4}$, V. Karsegard ${ }^{4}$, F.R. Herrmann ${ }^{5}$, C. Graf ${ }^{1}$, 1. Rehabilitation and palliative Care, University Hospitals of Geneva, Bernex,
Geneva, Switzerland, 2. Charite University Medicine Berline, Berlin, Germany, 3. Social and Preventive Medicine, University of Bern, Bern, Switzerland, 4. Clinical Nutrition, University Hospitals of Geneva, Geneva, Switzerland, 5. Geriatrics, University Hospitals of Geneva, Geneva, Switzerland

Phase angle measured by bioelectrical impedance analysis (BIA) may be a marker of health state. This study aims to investigate the link between phase angle and mortality in older persons, and to evaluate whether we can define a phase angle cut-off.

We included all adults over 65 years who underwent a BIA measurement at the Geneva University Hospitals between 1990 and 2011. We retrieved the phase angle and co-morbidities at the last BIA measurement and the mortality until December 2012. The analyses were performed with the Nutriguard ${ }^{\circledR}$ device because they allow the calculation of sex-, age- and body mass index (BMI)- standardized phase angle using German reference values. Sex-specific and standardized phase angle were categorized into quartiles, where quartile 1 corresponds to the lowest phase angle values and is used as reference category. We evaluated the association of mortality with sex-specific or standardized phase angle through univariate and multivariate (age, sex, comorbidities, BMI categories and settings) Cox regression models, KaplanMeier curves and ROC curves.

1307 (38\% women) underwent a measurement with the Nutriguard device. Death occurred in 628 persons $(44 \%$ women). In a multivariate Cox regression model, the risk of mortality decreased progressively as the standardized phase angle quartile increased (HR $0.71(95 \% \mathrm{CI} 0.58,0.86), 0.53$ (95\% CI $0.42,0.67), 0.32$ (95\% CI $0.23,0.43)$. The discriminative value of continuous phase angle, assessed as the area under the ROC curve, was $0.724(95 \% \mathrm{CI} 0.70,0.75)$ not leading to define an acceptable phase angle cut-off to perform individual prediction of mortality.

This study shows the association of phase angle and mortality in elderly patients, independently of age, sex, comorbidities, BMI categories and settings (ambulatory vs hospitalized).

\section{COMPARISON OF OLDER VS. YOUNGER HOSPITALIZED ADULTS WITH A MALNUTRITION DIAGNOSIS IN THE U.S., 2010}

R. Di Maria-Ghalili ${ }^{1}$, J. Slaughter ${ }^{1}$, E.W. Gonzalez ${ }^{1}$, P. Abeysekara ${ }^{1}$, H. Resnick ${ }^{2}$, P. Guenter ${ }^{3}, 1$. Drexel University, Philadelphia, Pennsylvania, 2. Resnick Chodorow LLC, Silver Springs, Maryland, 3. American Society for Parenteral and Enteral Nutrition, Silver Springs, Maryland

Purpose: To examine differences in demographic characteristics and comorbidities in a nationally representative sample of older ( $\geq 65$ years) and younger (18-64 years) hospitalized patients with diagnosis of malnutrition (dxmal).

Methods: Data are from the 2010 Healthcare Cost and Utilization Project (HCUP), which contain patient-level data with ICD-9CM diagnosis codes on hospital inpatient stays. Data were weighted to generate nationally representative estimates of US hospitalizations. Characteristics of older and younger adults with a dxmal during their hospital stay were identified and compared. 